Artículo

\title{
Análisis FODA en el proceso de autoevaluación de la carrera de Bachillerato en Informática Empresarial en la UCR, Sede de Occidente
}

SWOT analysis in the self evaluation process of the Bachelor of Business Computing career at the western campus of Universidad de Costa Rica

Denis González Herrera*

dennis.gonzalezherrera@ucr.ac.cr

Sindy Porras Santamaría*

sindy.porras@ucr.ac.cr

ISSN 1996-1642, Editorial Universidad Don Bosco, año 9, No.15, enero-junio de 2015, pp. 39-53

Recibido: 7 de octubre de 2014 . Aprobado: 10 de diciembre de 2014.

\section{Resumen}

El presente artículo presenta los resultados de la autoevaluación que, con miras a la acreditación, se realizó en la carrera de Bachillerato en Informática Empresarial en la Sede de Occidente de la Universidad de Costa Rica. Para eso se utilizó la técnica del FODA, ya que ésta permite conocer la situación inicial, facilita una mayor participación de las personas involucradas en el proceso, brinda la retroalimentación de la información a las distintas poblaciones, incentiva la autocrítica dentro de la Unidad Académica y suministra espacios de discusión. El identificar y analizar las principales fortalezas y debilidades de la carrera resultó fundamental en la autoevaluación ya que son insumos a tomar en cuenta en el mejoramiento continuo de la calidad de la educación superior en general y de la mencionada carrera en particular.

Palabras clave: autoevaluación, acreditación, FODA, Universidad de Costa Rica, Informática Empresarial.

\section{Abstract}

This paper shows the outcomes of the self-evaluation process that, looking forward to accreditation, was carried out in the Bachelor of Business Computing career at the western campus of Universidad de Costa Rica. In doing that, the SWOT analysis was used since it allows diagnosing the initial situation, promotes a wider participation of people in the process, provides feedback, encourages self-criticism, and provides opportunities for discussion. Identifying and analyzing the main strengths and weaknesses in the career are key elements in the self evaluation process as they provide raw materials to continuous improvements of education quality in general but particularly of this major.

Keywords: self evaluation, accreditation, SWOT, Universidad de Costa Rica business computer.

* Profesor del Departamento de Ciencias Naturales, Sede Occidente, UCR, Costa Rica.

** Directora del Departamento de Ciencias Naturales Sede Occidente, UCR, Costa Rica.

Para citar este artículo: González, D. y Porras, S. (2015). Análisis FODA en el proceso de autoevaluación de la carrera de Bachillerato en Informática Empresarial en la UCR, Sede de Occidente. Diá-logos 15, 39-53. 


\section{Introducción}

El presente artículo expone los resultados del Análisis de Fortalezas, Oportunidades, Debilidades y Amenazas (FODA), así como su utilización como herramienta en el proceso de autoevaluación de la carrera en Bachillerato en Informática Empresarial (IE). Se expone además cómo el modelo FODA permitió evidenciar aspectos internos y externos que influyen directamente en la calidad de la IE. De igual manera, se pretende que el lector conozca las particularidades que caracterizan la carrera y su camino al mejoramiento de la calidad, concentrando el estudio en la Sede de Occidente de la Universidad de Costa Rica.

En la primera parte se resaltan algunas nociones importantes sobre autoevaluación y el proceso de acreditación, permitiendo profundizar en los conceptos de calidad de la educación universitaria, así como también en la definición de FODA. La segunda parte destaca el contexto en que se ha venido desarrollando la IE, esto con la intención de exponer el contexto actual donde se desarrolla el estudio. En esta sección también se describe el desarrollo del análisis FODA al proceso de Autoevaluación en la Sede de Occidente. Finalmente se presenta los principales hallazgos y conclusiones relacionadas con la experiencia y la importancia de utilizar métodos que permitan identificar los aspectos internos y externos del ambiente académico con el propósito de evidenciar la situación actual de la organización educativa.

\section{Referentes conceptuales}

\section{Autoevaluación}

Reyes (2007) y Redon (2009) definen la autoevaluación como una herramienta para crear y aumentar la calidad de los programas e instituciones, al permitir identificar sus fortalezas y debilidades. Ellos conciben la autoevaluación como el logro de equilibrar los elementos internos y externos del ambiente educativo. Además, Thompson, Strickland, Colado y Carreón (1998) establecen que el análisis FODA estima el hecho que una estrategia tiene que lograr un equilibrio o ajuste entre la capacidad interna de la organización y su situación de carácter externo; es decir, las oportunidades y amenazas. Rectoría de la Universidad de Costa Rica (2011) al definir el proceso de autoevaluación, la entiende como la acción dentro del entorno formativo que busca captar información permanente sobre las limitaciones, debilidades, fortalezas, amenazas y oportunidades; que oriente de manera objetiva la toma de decisiones.

Análisis FODA en el proceso de autoevaluación de la carrera de Bachillerato en Informática Empresarial en la UCR, Sede

40. de Occidente

\section{Acreditación}

El principal objetivo de la acreditación es dar fe pública del cumplimiento con los estándares establecidos, tanto para las instituciones como para sus programas académicos (Lémez, 2005; Castro y Muñoz, 2004). Dentro de este contexto, la Red Iberoamericana de Acreditación de la Calidad de la Educación Superior (RIACES) manifiesta que las entidades deben ajustarse a criterios y estándares establecidos 
por la agencia u organismo acreditador. Se pone en evidencia contar en el entorno con organismos de arbitraje que garanticen los esquemas establecidos. Existen entidades de acreditación de la calidad o iniciativas orientadas a la creación de organismos nacionales de acreditación de la educación superior en la mayor parte de los países de América Latina y el Caribe que expresan la relevancia por lograr el aseguramiento de la calidad en la educación superior mediante el proceso de autoevaluación con fines de acreditación (Mora, 2005). Fernández y IESALC (2006) expone precisamente la lista de Organismos de Aseguramiento de la Calidad en América Latina y el Caribe.

La acreditación oficial en el ámbito costarricense es otorgada por el Consejo Nacional de Acreditación del Sistema Nacional de Educación Superior (SINAES). La acreditación constituye un proceso que comprende regularmente cuatro etapas, dieciocho fases y múltiples acciones. El Sistema Nacional de Acreditación de la Educación Superior (2009) resume las principales etapas de la siguiente manera: La primera etapa o etapa inicial corresponde a la información, motivación, sensibilización y reflexión interna de la comunidad académica de la carrera, la decisión consensuada de participación y el aval de las autoridades rectoras de la institución de educación superior.

La segunda etapa corresponde a la autoevaluación que realizan los actores involucrados en la carrera que se ha sometido al proceso de acreditación: académicos, investigadores, administradores, estudiantes, egresados, colegios profesionales universitarios y empleadores, entre otros.

La tercera etapa del proceso de acreditación oficial corresponde a la evaluación externa, cuyos propósitos son: por un parte, la validación que realizan pares académicos externos internacionales tanto de la etapa de autoevaluación como del informe resultante de ésta y por otra parte, la evaluación directa e in situ de la carrera o programa.

La cuarta etapa o etapa de acreditación y mejoramiento continuo comprende la valoración final de la calidad de la carrera que realiza el Consejo Nacional de Acreditación del (SINAES) mediante un proceso de triangulación que considera (1) el Informe Final de Evaluación Externa, (2) el Informe de Autoevaluación, (3) el Compromiso de Mejoramiento y (4) toda otra información obtenida a lo largo de las diversas etapas, fases y actividades del proceso. Finalmente, comprende también, la decisión indelegable del Consejo del SINAES de conceder o no la acreditación oficial. Cabe destacar que actualmente la IE se encuentra en una intersección entre las etapas segunda y tercera ya mencionadas.

\section{Calidad de la educación superior}

El Sistema Nacional de Acreditación de la Educación Superior (2009) es la instancia que establece las políticas sobre calidad las cuales expresan: Establecer, mantener y fortalecer permanentemente un sistema de mejoramiento continuo 
de la calidad, al igual que un sistema de evaluación, valoración y administración de riesgos de los procesos. Incentiva la permanente autoevaluación mediante procesos participativos y críticos en áreas relativas y específicas, promueva la actualización en el conjunto de herramientas necesarias para el cumplimiento de la misión educativa y por último propicia la realimentación y aprendizaje de todos los involucrados en el quehacer universitario.

Como señala Dias Sobrinho (1995), el concepto de calidad es una construcción social, que varía según los intereses de los grupos de dentro y de fuera de la institución educativa que refleja las características de la sociedad actual y que se proyecta para el futuro. El concepto de calidad es una idea de construcción colaborativa y gradual que integra, articula visiones, y propicia demandas diferentes con los valores y propósitos de la institución educativa Fernández y IESALC (2006).

\section{FODA}

Procede del acrónimo en inglés SWOT (Strengths, Weaknesses, Opportunities, and Threats), en español las siglas son FODA (Fortalezas, Oportunidades, Debilidades y Amenazas). Talancón (2006) describe al análisis FODA como una herramienta que permite diseñar la situación actual de la empresa en función de estos cuatro criterios realizando una evaluación de los aspectos fuertes y débiles del ambiente interno de la organización, lo cual brinda la posibilidad de actuar en función de ellas. En contraparte, las oportunidades y amenazas son externas por lo que resulta difícil poder modificarlas. También es una metodología simple que permite capturar una perspectiva global de la situación estratégica de una determinada organización.

Este tipo de análisis permite realizar diagnósticos en las organizaciones laborales es una condición para intervenir profesionalmente en la formulación e implantación de estrategias y su seguimiento para efectos de evaluación y control (Talancón, 2006; Olivera y Hernández, 2011).

Según Navarro (1998) es conveniente y enriquecedor el trabajar con el análisis FODA, el cual se refiere a detectar desde lo interno aspectos fuertes y positivos en la gestión institucional así como también aspectos débiles y negativos; y desde lo externo, los aspectos favorecedores para la gestión y aspectos perjudiciales del entorno.

\section{Bachillerato en informática empresarial}

El bachillerato en Informática Empresarial es una carrera universitaria que se imparte en la Universidad de Costa Rica desde el año 1998 (Universidad de

Análisis FODA en el proceso de autoevaluación de la carrera de Bachillerato en Informática Empresarial en la UCR, Sede

42. de Occidente Costa Rica, 1997) tanto en la sede central como en todas las Sedes Regionales. La carrera se imparte en el Recinto de Golfito, Sede del Atlántico, Recinto de Paraíso, Recinto de Turrialba, Sede de Guanacaste, Sede de Limón, Sede de Occidente, Recinto de Grecia y Sede del Pacífico. Lo que cubre la oferta académica en la totalidad del país (Chaves y Ramírez, 2012) 
Como lo indica el nombre de la carrera, se le da un enfoque empresarial, lo que quiere decir que en su plan de estudios cuenta con cursos del área de administración de negocios, que la hace diferente a las otras opciones de carreras de informática que se brindan en el país. Según Chaves y Ramírez (2012), la formación del informático empresarial se construye a partir de tres áreas del conocimiento: la computación, la informática y la administración, con el apoyo de la matemática y la lógica, teniendo como ejes la ética y el humanismo. Este profesional está capacitado para analizar, diseñar y programar sistemas, utilizando tecnología de punta, así como para la planificación, control y dirección de la gestión informática en la empresa o institución. Además, es capaz de formar parte activa en trabajos complejos y para dirigir investigaciones multidisciplinarias aplicadas.

La demanda de los estudiantes por ingresar a la carrera es muy alta, tanto así que son insuficientes los cupos de nuevo ingreso que se abren año con año en todos los lugares en donde se imparte. El profesional graduado de nuestra carrera es altamente cotizado a nivel laboral por su perfil y muchos comienzan a trabajar incluso antes de graduarse.

Específicamente en la Sede de Occidente, la carrera es parte del Departamento de Ciencias Naturales y se imparte en los recintos de San Ramón y Grecia, donde cada año se abre una promoción con un promedio de treinta y cinco estudiantes aceptados en cada una. Además, se cuenta con un grupo de dieciséis profesores, entre interinos y propietarios, por ciclo lectivo para cubrir ambos recintos y solamente un encargado de carrera.

Para el primer ciclo lectivo del año 2013, al mes de marzo, en la carrera se contabilizan doscientos veintidós estudiantes activos en la carrera IE en el Recinto de San Ramón y ciento noventa y ocho en el Recinto de Tacares según el Sistema de Aplicaciones Estudiantiles.

\section{Método}

\section{El proceso de autoevaluación}

El proceso de autoevaluación de la carrera de IE inicia en el año 2009 cuando el Consejo de Carrera, integrado por los encargados de coordinarla en todas las unidades académicas donde se imparte, acuerda optar por una acreditación a nivel nacional y se cuenta con el respaldo del Consejo de Sedes. Dicha acreditación la brinda el Sistema Nacional de Acreditación de la Educación Superior. Órgano oficial de acreditación de la educación superior de Costa Rica, es de carácter nacional y fue creado por Ley de la República No 8256 del 2 de mayo de 2002. 
Para iniciar este proceso cada Unidad Académica designa a un representante de la carrera para formar una comisión que se encargaría de la autoevaluación y acreditación. Dicha comisión se denomina Comisión Integradora (Cl) y se mantiene vigente hoy en día, trabajando por conseguir los objetivos. Algunos miembros han cambiado con el paso del tiempo. En el caso específico de la Sede de Occidente inicialmente se designó a una persona para el proceso, pero actualmente tres profesores de la carrera cuentan con jornada para este fin. Además, la sede cuenta con una comisión que está integrada por profesores de la carrera, funcionarios administrativos y estudiantes, que colaboran en la recopilación de las evidencias para el proceso de autoevaluación, y está liderada por los representantes ante la $\mathrm{Cl}$.

Cada unidad académica realizó a nivel interno la autoevaluación de la carrera con la meta de concluir esa etapa en el presente año (2013) para posteriormente optar por la acreditación. Para eso se siguió las especificaciones del Sistema Nacional de Acreditación de la Educación Superior (2009) y sus cuatro etapas del proceso de acreditación, iniciando con la información, motivación, sensibilización y reflexión interna de la comunidad académica de la carrera. Se continuó con la decisión consensuada de participación y el aval de las autoridades rectoras de la institución de educación superior. Posteriormente se realizó la autoevaluación con los actores involucrados en la carrera, para continuar con la evaluación externa. Por último se asumió la etapa de acreditación y mejoramiento continuo que la institución y el seguimiento por parte del SINAES con las obligaciones contraídas en el Compromiso de Mejoramiento, cuyo objetivo era y es, superar las debilidades encontradas y enriquecer las fortalezas.

El proceso de autoevaluación buscaba determinar las deficiencias y establecer las mejoras en la calidad de la carrera. Como se indica en Civit (2001), evaluar una institución es una tarea que involucra su razón de ser, su trayectoria histórica y su destino. La evaluación en una institución universitaria es un proceso que acompaña su actuación diaria, refleja sus resultados e ilumina la toma de decisiones. Esto permite su puesta al día y mejora constantes en el corto plazo y orienta las transformaciones de fondo y reorganizaciones estructurales para el mediano y largo plazo. En este sentido, la evaluación de la universidad forma parte de su proceso de renovación permanente.

Según Martínez (1997) la acreditación es un mecanismo para determinar el nivel global de una institución de educación superior, una "certificación" de la calidad académica de un programa o unidad, o un juicio sobre la consistencia entre los objetivos, los recursos y la gestión de una unidad académica. El proceso de

Análisis FODA en el proceso de autoevaluación de la carrera de Bachillerato en Informática Empresarial en la UCR, Sede 44. de Occidente Acreditación suele comprender la autoevaluación, la evaluación externa y el informe final.

La carrera de IE tiene la particularidad que fue creada por varias sedes, por lo que le pertenece a todas a la vez. Esto significa que, al menos teóricamente, 
la carrera es la misma en todos los lugares en dónde se imparte, y cualquier cambio o modificación debe estudiarse y ser aprobado por todas las unidades implicadas.

Esto ha venido a ser un reto a nivel del proceso de autoevaluación, ya que, debido a que las sedes se encuentran geográficamente distantes, las particularidades de la ubicación de la unidad académica han hecho que algunas cosas sean diferentes. Por mencionar sólo un ejemplo, en el curso de Práctica Empresarial los estudiantes de la Sede de Limón realizan proyectos enfocados a las empresas de la zona que son más de corte marítimo y portuaria, mientras que los estudiantes de la Sede de Occidente realizan sus proyectos en empresas del Valle Central, algunas de ellas dedicadas al desarrollo de software. Algunas de las diferencias se han ido subsanando en la marcha, otras quedarán para realizarse como compromiso de mejora una vez acreditada la carrera.

\section{Metodología}

El enfoque de investigación utilizado como base para abordar la problemática estudiada es el enfoque cualitativo de tipo descriptivo. Se hace referencia además al diseño de estudio, a la población abordada, así como a las técnicas de recolección de datos y de análisis de la información.

\section{Diseño}

Se utiliza el enfoque de investigación cualitativo, con el propósito de obtener una perspectiva del estado del proceso de autoevaluación, haciendo uso de distintas fuentes de información.

A partir de dicho enfoque se logran comprender las fortalezas, oportunidades, debilidades y amenazas del proceso que permite, a través del análisis de los datos, que generan resultados para ser utilizados en el informe final de autoevaluación y en la elaboración de los objetivos de mejora para la carrera de IE.

En general, la investigación se lleva a cabo con un propósito descriptivo e interpretativo, que permite exponer cómo el modelo FODA pone en evidencia aspectos internos y externos que influyen directamente en la calidad de la IE en su camino al mejoramiento continuo mediante los procesos de autoevaluación.

Características del área y población en estudio

La Universidad de Costa Rica cuenta con cinco Sedes regionales (Atlántico, Guanacaste, Pacífico, Limón y Sede de Occidente) todas se encuentran realizando en conjunto el proceso de autoevaluación para la carrera de Bachillerato en informática empresarial.

Para efectos del planteamiento se seleccionó la Sede de Occidente, que abarca el Recinto de San Ramón y el Recinto Universitario de Grecia. 
La carrera en esta sede contabilizó al 2013 un total de doscientos veintidós estudiantes activos y ciento noventa y ocho estudiantes respectivamente. Además, se cuenta un cuerpo docente de dieciséis personas que atienden ambos recintos.

Muestra

Se utilizó la información recopilada desde el año 2009 en que inició el proceso de autoevaluación y las evidencias completas, específicamente de la Sede de Occidente, que aproximadamente corresponden al setenta por ciento de un total de 348 evidencias solicitadas según los requerimientos del SINAES.

Métodos, técnicas e instrumentos de recolección de datos Se realizó una revisión de las evidencias recolectadas de las diferentes fuentes y actores. Además, se basó en la experiencia adquirida a lo largo del proceso de autoevaluación como miembros de la Cl. La información fue categorizada en fortalezas, oportunidades, debilidades y amenazas siguiendo el análisis FODA. Además, se recolectaron referencias bibliográficas que fundamentan los antecedentes y las referencias conceptuales.

Proceso de análisis de la información El análisis de datos se basó en el marco teórico, la información recopilada como evidencias de autoevaluación y la revisión bibliográfica, tanto de los datos de la carrera de IE, como lo que tiene que ver con el proceso de acreditación por medio de SINAES.

El análisis se realizó de acuerdo a los factores externos (fortalezas y debilidades) y los factores internos (oportunidades y amenazas), que sirvieron como material para completar el FODA y obtener los resultados que aquí se muestran.

\section{Resultados del análisis FODA en el proceso de autoevaluación}

Como una forma de darle a conocer a las autoridades de la Sede de Occidente y a los estudiantes de la carrera el avance y las dificultades que se han presentado en el proceso de autoevaluación, se decidió realizar un análisis FODA. Basándonos en la experiencia desarrollada en este proceso, como miembros de la $\mathrm{Cl}$ y de la Comisión de Autoevaluación de la carrera y en los resultados obtenidos hasta el momento en la recolección de evidencias y preparación del informe. Los resultados fueron expuestos a la comunidad universitaria involucrada mediante una presentación formal. En dicho análisis se detectaron las siguientes fortalezas, oportunidades, debilidades y amenazas.

Análisis FODA en el proceso de autoevaluación de la carrera de Bachillerato en Informática Empresarial en la UCR, Sede

46. de Occidente

\section{Fortalezas}

1. Lo que más ha repercutido es la creación de una comisión de trabajo integrada por representantes de todas las unidades académicas de la UCR 
donde se imparte la carrera de IE: Recinto de Golfito, Sede del Atlántico, Recinto de Paraíso, Recinto de Turrialba, Sede de Guanacaste, Sede de Limón, Sede de Occidente, Recinto de Grecia y Sede del Pacífico.

2. Desde el inicio del proceso de autoevaluación de la carrera este grupo de personas, denominado Comisión Integradora, ha venido trabajando en el entendimiento del proceso, la recolección de evidencias, la redacción del informe, la revisión de las encuestas y la elaboración de los compromisos de mejoras.

3. Al contar con miembros de todas las sedes y recintos conocedores de la carrera en cada una, siendo algunos profesores, otros encargados de la carrera en su unidad de trabajo, estudiantes y asistentes, se ha logrado tener una visión global, encontrando las diferencias y semejanzas de la misma en donde se imparte.

4. El proceso fue pensado con miras de acreditación ante el SINAES, pero antes de ser presentado a dicho ente, debe pasar por la revisión y aprobación del Centro de Evaluación Académica (CEA) de la UCR. Esta instancia ha estado presente en el proceso desde sus inicios, colaborando de manera activa y dando las pautas a seguir, por medio de la colaboración de una de sus funcionarias y con el apoyo de otras personas expertas en diferentes procesos.

5. Como parte de este proceso se realizan reuniones continuamente, tanto presenciales en el CEA, como en encerronas de trabajo de dos o tres días en la Sede del Pacífico. En éstas últimas se han obtenido resultados muy favorables para el proceso, ya que se les da la oportunidad a más profesores de todas las Sedes a participar y a aportar sus conocimientos sobre la carrera. Incluso algunos de ellos fueron parte de su creación, y sus experiencias han enriquecido la recolección de evidencias.

6. Por otro lado la carrera, en cada unidad académica donde se imparte, tiene su propia comisión de autoevaluación, las cuales están formadas por profesores, estudiantes y funcionarios administrativos; que colaboran con la recolección de las evidencias que solicita el SINAES para la presentación del Informe de Autoevaluación.

7. Específicamente en la Sede de Occidente, por estar compuesta por dos recintos, San Ramón y Grecia, se conformó una sola comisión con miembros de ambos lugares. Ésta ha variado con el paso de los años, ya que algunos de los estudiantes que iniciaron el proceso ya se graduaron y ahora son egresados, y algunos funcionarios administrativos han cambiado de puestos. Sin embargo, la comisión se ha mantenido con la ayuda de los profesores de la carrera, quienes se han demostrado su compromiso. 
8. Además, en la Sede de Occidente, se cuenta con estudiantes becados quienes realizan sus horas de trabajo en la ayuda a los encargados de autoevaluación en la recolección de evidencias. Algunos de los estudiantes egresados que fueron parte de la comisión también colaboran con el proceso desde su perspectiva de profesionales graduados de la carrera.

9. Una fortaleza muy importante ha sido los conocimientos tecnológicos de los miembros de la Cl y de las comisiones de las sedes, ya que al ser en su mayoría informáticos ha beneficiado el uso de herramientas tecnológicas en el desarrollo del proceso, como por ejemplo las videoconferencias y los archivos compartidos.

10. Al ser un trabajo arduo que requiere de mucha organización para cumplir con los tiempos de entrega y recolección de evidencias, se ha desarrollado un cronograma de trabajo que se actualiza con el pasar del tiempo, y en el que se contemplan las fechas de reuniones, plazos de recolección de evidencias, aplicación de encuestas, redacción del informe y los responsables de cada actividad.

\section{Oportunidades}

1. El proceso ha permitido obtener una visión global de la carrera de IE tanto a nivel de la Sede de Occidente como en los demás sitios en donde se imparte. Las diferentes reuniones y encerronas de trabajo de la $\mathrm{Cl}$ y de los profesores de todas las sedes y recintos, han dado como resultado el conocimiento general del comportamiento de la carrera en cada una de ellas, las semejanzas y diferencias según el lugar.

2. Gracias a esto, algunas de las diferencias fueron ya eliminadas y se han estandarizado procesos y funciones, como por ejemplo los programas de cursos, el formato de los mismos y su nivel de dificultad. Esto garantiza que se mantenga la misma complejidad y calidad de la carrera en todos los lugares en donde se imparte.

3. Como parte del proceso se requiere realizar encuestas a las personas relacionadas con la carrera de manera directa: profesores, estudiantes, funcionarios administrativos, graduados y empleadores. Una vez que se cuente con la información que ellos brinden se tendrá una visión más amplia del panorama de la carrera y de la valoración que se le da tanto a lo interno de la Universidad como en la sociedad.

4. Otra oportunidad encontrada es que una vez finalizado el proceso de autoevaluación el siguiente paso es la presentación del informe antes el SINAES

Análisis FODA en el proceso de autoevaluación de la carrera de Bachillerato en Informática Empresarial en la UCR, Sede

48. de Occidente para optar por la acreditación. Este informe será presentado a nivel de toda la carrera, contemplando cada sede y recinto por separado, y posteriormente, esta entidad evalúa el informe y determina cuáles unidades académicas califican para obtener la acreditación de la carrera. 
5. El fin que se tiene en la Sede de Occidente es obtener la acreditación de la carrera, lo que además garantiza mejoras significativas, ya que se estaría avanzando en varias dimensiones, según los componentes evaluados de acuerdo con los criterios del SINAES, entre ellos: plan de estudios, personal académico, infraestructura, equipo y materiales, desarrollo docente, metodología de enseñanza y materiales, por mencionar solo algunos.

\section{Debilidades}

1. El proceso de autoevaluación conlleva una carga de trabajo grande, ya que se deben recolectar trescientas cuarenta y ocho evidencias que solicita el SINAES, posteriormente la redacción del informe y los compromisos mejora. Esta es una labor que implica mucho tiempo, traslados a reuniones, búsqueda de información en diferentes instancias administrativas, aplicación de encuestas, comisiones y encerronas de trabajo, análisis de datos, entre otros.

2. Cuando se tomó la decisión de iniciar con la autoevaluación se nombró a una persona por cada sede y del Recinto de Golfito. Este nombramiento ha sido por un octavo de tiempo cada semestre, lo que significa cinco horas semanales, con apoyo de la Vicerrectoría de Docencia de la UCR. Sin embargo, debido a la cantidad de trabajo que representa este proceso, y a que en algunos casos un sede cuenta con más de un recinto, como es el caso de la Sede de Occidente que tiene el Recinto Universitario de Grecia, el trabajo se duplica o triplica, por lo que el tiempo asignado se vuelve insuficiente.

4. Las particularidades de cada sede han dificultado el poner de acuerdo a todos los miembros de la comisión. Como cita Saíz y Gómez (2007) las dificultades y problemas inherentes a la evaluación de la actuación o ejecución de los sujetos que trabajan en grupo cooperativo han sido puestas de manifiesto por trabajos como los de King y Behnke (2005), quienes destacan cómo las mismas se convierten en factores que dificultan la puesta en práctica de esta estrategia colaborativa.

5. En el caso específico de la Sede de Occidente, en los últimos ciclos lectivos se ha contado con la ayuda del Departamento de Ciencias Naturales y de la Coordinación de Docencia para asignar jornada de la Unidad Académica para este propósito. Esta jornada ha variado con el tiempo, según las necesidades que existan y la demanda de cursos, por lo que no es fija ni se tiene garantizada, y el tiempo invertido ha ido desde un octavo de tiempo hasta tres tiempos completos en el tercer ciclo del 2012.

6. Esta situación no es igual en las demás sedes. La Cl ya ha realizado gestiones ante el Consejo de Área para que esta jornada se amplíe en todas las sedes y recintos dada la necesidad de terminar pronto con el informe y que no se desactualice la información que ya ha sido recopilada. Estas gestiones no han rendido fruto hasta el momento. 
7. Por otro lado, en la etapa de recolección de evidencias se requiere de la ayuda de todos los profesores de la carrera, estudiantes y administrativos, y dado que estos no cuentan con tiempo asignado para ello se han presentado situaciones de atrasos en los tiempos de entrega, ya que no lo toman como una prioridad, sobre todo los funcionarios administrativos que tienen múltiples funciones y no cuentan con tiempo suficiente para dedicarlo a contestar los oficios de solicitud de información.

8. Además, no se logrado realizar una campaña ardua de sensibilización de la población universitaria, aunque se han tomado algunas acciones como pancartas explicativas y presentaciones a los estudiantes de la carrera, todavía falta mejorar en este tema.

\section{Amenazas}

1. La carrera de IE es la primera en realizar un proceso de autoevaluación con la particularidad de que es una carrera que le pertenece a todas las sedes por igual, lo que, hasta el momento, es un caso único en la UCR, y que implica que para el CEA no cuente con experiencia previa para guiar este proceso en específico.

2. Además, el proceso de autoevaluación ha requerido de mucho tiempo en todas las sedes y recintos, debido a las debilidades antes indicadas, y eso hace que muchas de las evidencias recolectadas pierdan vigencia, ya que tienen un máximo de 6 meses de antigüedad al momento de entregar el informe ante el SINAES, lo que implica que hay que volver a realizar el trabajo para actualizarlas.

3. El avance de la recopilación de evidencias ha sido distinto en todas las unidades académicas, lo que ha generado que se amplíen los plazos de finalización del informe y presentación del mismo ante el CEA para su revisión.

\section{Conclusiones}

Al ser una carrera con la particularidad de pertenecer a varias sedes a la vez el reto de conseguir la acreditación ante el SINAES es diferente que para el resto de las carreras de la UCR. Esto implica una mayor organización y trabajo por parte de la $\mathrm{Cl}$ y de las comisiones a nivel interno de las unidades académicas.

La matriz FODA como herramienta viable para realizar análisis organizacional, en relación con los factores que determinan el éxito en el cumplimiento de metas, es una alternativa que motivó a efectuar el análisis para su difusión y divulgación. Con él se logró determinar las fortalezas, oportunidades, debilidades y amenazas

Análisis FODA en el proceso de autoevaluación de la carrera de Bachillerato en Informática Empresarial en la UCR, Sede

50. de Occidente encontradas durante la participación en el proceso de autoevaluación de la carrera de IE en la Sede de Occidente de la UCR.

Entre las principales fortalezas encontradas está el trabajo en equipo de la Cl, la cual ha trabajado desde el 2009 en poner en práctica el proceso de autoevaluación, 
con el objetivo de buscar la mejora constante en la calidad de la carrera, y la obtención de la acreditación por parte del SINAES. El aporte de la comisión de la sede, los docentes, estudiantes y personal administrativo ha fortalecido también esta etapa. La colaboración de todos los involucrados ha sido fundamental en la recolección de evidencias para la elaboración del informe.

Como resultado de este análisis, una de las oportunidades que se ha detectado son las diferencias existentes entre las sedes entorno a la IE y que algunas de ellas se han logrado corregir y unificar criterios durante la autoevaluación. Esto ha generado mejoras visibles, incluso antes de terminar el proceso y de presentar el informe.

A consecuencia de este estudio se identificó que la magnitud del trabajo que representa este proceso es grande, la recolección de evidencias es un procedimiento compendioso que depende, en la mayoría de los casos, de la información que las diferentes oficinas puedan suministrar. El apoyo de jornada para realizar estas labores ha sido insuficiente en el pasado, aunque actualmente la sede brinda una mayor ayuda en este aspecto, pero no ha sucedido lo mismo en las demás sedes. Lo que ha significado que el avance varíe.

Lo anterior repercute en una amenaza, ya que se han presentado atrasos en las fechas definidas en el cronograma establecido por la $\mathrm{Cl}$ justamente por los desfases en la recolección de evidencias entre las diferentes sedes, y esto en muchos casos hace que las evidencias recolectadas pierdan vigencia y deban ser nuevamente recolectadas, lo que representa un trabajo doble.

Aun cuando se presentan varias debilidades y amenazas, hay que resaltar que las fortalezas y oportunidades hacen ver que el proceso va bien encaminado y que la meta a lograr está en proceso a cumplirse en un plazo cercano.

Con la ayuda del análisis FODA se ha podido dar a conocer a las autoridades de la Sede de Occidente, a los profesores y estudiantes de la carrera la situación actual del proceso de autoevaluación, y se han identificado los puntos débiles que deben ser valorados para mejorarlos y que no se conviertan en una traba para concluir satisfactoriamente con la acreditación.

\section{Recomendaciones}

Se recomienda realizar este tipo de estudios en las demás sedes y recintos donde se imparte la carrera a fin de tener una visión global del proceso de autoevaluación. Esa información además serviría como herramienta para informar a las autoridades del estado actual de cada una, al Consejo de Sedes y a la Vicerrectoría de Docencia sobre el avance del mismo.

Asimismo, es importante dar a conocer los resultados de este análisis a las nuevas generaciones de estudiantes de la carrera, para que conozcan el 
trabajo realizado hasta el momento, entiendan la importancia de llevar a cabo la acreditación y de los beneficios que como futuros profesionales obtendrán. Además, de incentivarlos a participar del proceso.

Por otro lado, es importante realizar a corto plazo nuevamente este análisis FODA en la Sede de Occidente, una vez concluido el proceso de autoevaluación, con el propósito de actualizar los datos y estar preparados para cuando el SINAES realice las evaluaciones respectivas.

También, al obtener una retroalimentación de todos los actores mediante las encuestas que serán aplicadas en los meses próximos se logrará tener un panorama integral de la carrera tanto a nivel interno del centro educativo, como a nivel de la sociedad. Lo que dará más materia para un siguiente FODA.

\section{Referencias}

Castro, J. L., y Muñoz, S. B. (2004). Reunión sobre propuestas de acreditación para Carreras de Farmacia de América Latina (Buenos Aires, 12 al 14 de Mayo, 2004). Documento base de discusión. Recuperado en 13 de enero de 2013, de http:// www.paho.org/Spanish/ad/ths/ev/sistemas-acreditacion-america-latina.doc

Chaves, M. A., y Ramírez, I. R. Escogencia de carrera y expectativas laborales según género para estudiantes del Bachillerato en Informática Empresarial de la Sede Occidente de la Universidad de Costa Rica. Presentado en Conferencia Latinoamericana de Informática, CLEl 2012 XXXVIII Conferencia Latinoamericana. En (pp. 1-10). IEEE.

Civit, J. (2001). Síntesis de enfoques y experiencias en evaluación institucional universitaria. Documento. Caracas: Universidad Católica Andrés Bello, Instituto de Investigaciones Económicas y Sociales.

Dias Sobrinho, J. (1995). Avaliação institucional, instrumento da qualidade educativa: a experiência da Unicamp. Avaliação Institucional-Teoria e experiências. São Paulo: Ed. Cortez.

Fernández Lamarra, N., y IESALC. (2006). La evaluación y la acreditación de la calidad: Situación, tendencias y perspectivas. Informe sobre la educación superior en América Latina y el Caribe 2000-2005: La metamorfosis de la educación superior. Recuperado en 8 de marzo de 2013, de http://estatico.uned.ac.cr/ paa/pdf/Informe_ESALC.pdf

Lémez, R. (2005). La acreditación de la educación superior en el Uruguay. Montevideo: UNESCO IESALC.

Martínez, E. (1997). La evaluación de la educación superior. Evaluación y acreditación universitaria. Metodologías y experiencias, Caracas, Nueva Sociedad. (p. 75-84). Recuperado en 7 de mayo de 2013, de http://www.unesco.org.uy/stmanagement/publicaciones/eval-em.htm.

Análisis FODA en el proceso de autoevaluación de la carrera de Bachillerato en Informática Empresarial en la UCR, Sede

52. de Occidente 
Navarro, A. M., y Ana, M. (1998). La autoevaluación de la gestión. CONEAU, Buenos Aires. Recuperado en 5 de mayo de 2013, de http://www.coneau.edu.ar/ archivos/1327.pdf.

Olivera, D., y Hernández, M. (2011). El análisis DAFO y los objetivos estratégicos. Contribuciones a la Economía, marzo 2011. Recuperado en 22 de abril de 2013, de http://www.eumed.net/ce/201 1a/domh.doc

Rectoría Nacional Dirección Nacional de Planeación. (2011). Modelo de autoevaluación y autorregulación con fines de mejoramiento y acreditación. (pp. 16-18). Bogotá: Ed. Kimpres.

Redon Pantoja, Silvia. (2009). Auto evaluación institucional y acreditación como aseguramiento de la calidad de la educación: Implicancias teóricas y prácticas. Estudios pedagógicos (Valdivia), 35(2), (pp. 269-284). Recuperado en 20 de marzo de 2013, de http://www.scielo.cl/scielo.php?script=sci_arttext\&pid=s0718$07052009000200016 \&$ Ing =es\&tIng=es. 10.4067/S0718-07052009000200016.

Reyes, S. Á. R. (2007). La autoevaluación de la carrera, una vía para el mejoramiento de la calidad académica. Revista Habanera de Ciencias Médicas, 6(3), (pp. 113). Recuperado en 07 de enero de 2013, de http://www.redalyc.org/articulo. oa?id $=180417641006$

Sáiz, M. S. I., y Gómez, G. R. (2007). El trabajo colaborativo en las aulas universitarias: reflexiones desde la autoevaluación. Revista de educación 344, 229-230. Recuperado en 7 de mayo de 2013, de http://www.revistaeducacion.mec.es/ re344/re344_15.pdf

Sistema Nacional de Acreditación de la Educación Superior (SINAES). (ED). (2009). Manual de Acreditación Oficial de Carreras de Grado del Sistema Nacional de Acreditación de la Educación Superior. San José, Costa Rica. Recuperado en 3 de enero de 2013, de http://www.sinaes.ac.cr/images/docs/proceso_ acreditacion/manual_grado_09.pdf.

Talancón, H. P. (2006). La matriz FODA: una alternativa para realizar diagnósticos y determinar estrategias de intervención en las organizaciones productivas y sociales. Contribuciones a la Economía, (2006-09).

Thompson, A. A., Strickland, A. J., Colado, P. I., y Carreón, M. A. S. (1998). Dirección y administración estratégicas: conceptos, casos y lecturas. México: Ed. McGraw-Hill.

Universidad de Costa Rica, Consejo de Carrera. (1997). Carrera de Bachillerato en Informática Empresarial. San José, Costa Rica. (pp. 46-154)

Universidad de Costa Rica, Sistema de Aplicaciones Estudiantiles (SAE). Fecha de acceso: 20 de marzo del 2013 